\title{
Échange et atmosphère d'écoute dans la pratique contemporaine du conte oral en France
}

\section{Émilie Rossignol}

\section{(2) OpenEdition \\ 1 Journals}

Édition électronique

URL : https://journals.openedition.org/clo/1711

DOI : $10.4000 /$ clo. 1711

ISSN : 2266-1816

Éditeur

INALCO

\section{Édition imprimée}

Date de publication : 2 janvier 2012

ISBN : 978-2-85831-212-2

ISSN : 0396-891X

\section{Référence électronique}

Émilie Rossignol, «Échange et atmosphère d'écoute dans la pratique contemporaine du conte oral en France », Cahiers de littérature orale [En ligne], 72 | 2012, mis en ligne le 29 avril 2015, consulté le 01 juillet 2021. URL : http://journals.openedition.org/clo/1711 ; DOI : https://doi.org/10.4000/clo.1711

Ce document a été généré automatiquement le 1 juillet 2021.

\section{(c) (i) (9)}

Cahiers de littérature orale est mis à disposition selon les termes de la Licence Creative Commons Attribution - Pas d'Utilisation Commerciale 4.0 International. 


\title{
Échange et atmosphère d'écoute dans la pratique contemporaine du conte oral en France
}

\author{
Émilie Rossignol
}

1 Depuis les années 1970-1980, en France, le phénomène de Renouveau du conte a fait émerger une pratique de l'oralité ayant des enjeux nouveaux. Les actes publiés sous la direction de Geneviève Calame-Griaule, suite aux rencontres sur ce thème en attestent et en présentent les aspects et questionnements (Calame-Griaule, 1999). Différents éléments ont permis ce renouveau, notamment les initiatives des bibliothécaires et le mouvement d'éducation populaire (Touati, 2000,12). Rappelons qu'une des missions des bibliothécaires consiste à valoriser le patrimoine oral, et qu'une des missions de l'éducation populaire, à partir du conte oral, permettrait de s'approprier ou de se réapproprier la parole, qu'elle soit ordinaire ou non.

2 Nous avons peu à peu assisté à la mise en spectacle du conte oral, surtout dans des théâtres et sur d'autres scènes. L'image du conte lors de veillées au coin du feu, même si elle peut faire émerger une certaine nostalgie à sa simple évocation, n'a pas pour autant disparue, mais cette pratique s'est considérablement modifiée.

Depuis presque quarante ans, les acteurs du renouveau du conte oral cherchent à légitimer cette pratique comme un art et une pratique artistique s'insérant dans une démarche à la fois globale, nationale et locale auprès de populations des plus diverses. En parallèle, cette pratique touche des milieux professionnel ou amateur, des enfants et des adultes, de l'initié au novice. Nous pouvons donner l'exemple de trois réseaux constitués :

- Mondoral, qui rassemble quatre structures œuvrant pour les arts de la parole: Paroles Traverses, la Maison du Conte, le Centre de Littérature Orale, le centre des Arts du Récit ${ }^{1}$,

- l'APAC, Association professionnelle des artistes conteurs ${ }^{2}$,

- le RNCAP, Réseau national du Conte et des Arts de la parole ${ }^{3}$. 
Ces trois réseaux œuvrent pour la reconnaissance et la légitimation du conte et des arts de la parole. Cette question a particulièrement été abordée lors des dernières rencontres nationales qui ont eu lieu à la Cartoucherie de Vincennes fin octobre 2012. ${ }^{4}$.

5 La pratique du conte oral ne semble pas statique depuis son renouveau et parait chercher à s'adapter à des contextes sociétaux, culturels et structurels changeants. Elle est bien vivante: des réseaux de conteurs, de professionnels du conte, etc., se sont développés et aujourd'hui, un public, notamment adulte, s'est constitué (Touati, 2000, 89 ; Didier, 1994, 2642).

6 Nous nous interrogerons ici sur le public, dans sa relation avec le conte et le conteur. Il apparaît que le public (Esquenazi, 2003), que les spectateurs, ne sont pas de simples réceptacles à histoires, mais qu'un ensemble d'éléments se révèlent et sont à l'œuvre lors de l'écoute des contes. C'est ce que nous proposons d'aborder au cours de cet article, dans la mesure où le public, les spectateurs, participent à cette pratique, car sans sa présence, un artiste pratiquant un art vivant serait difficilement concevable.

Nous nous baserons sur nos données de terrain ${ }^{5}$, observations et entretiens, majoritairement réalisés en région Rhône-Alpes, afin de mettre en évidence ce qui peut se jouer ou être à l'œuvre lors de l'écoute, pratique d'apparence passive.

Nous avons pu constater que le conte oral ne se présente seul que de façon exceptionnelle. En effet, il mêle ou croise d'autres genres appartenant à la littérature orale : le chant, les proverbes, les devinettes, les anecdotes, de la musique, de la danse et/ou des poèmes peuvent également accompagner le conte et/ou le conteur 6 .

Mentionner ces éléments nous permet d'envisager la pratique du conte oral comme une interaction sociale qui met en présence différents acteurs agissant dans un contexte particulier, spécifique : le conteur s'adresse à un public. Nous garderons à l'esprit le concept de performance, qui a été développé dans les années 1970 en ethnolinguistique et notamment défini par Richard Bauman (1975). Il nous permet de placer notre analyse dans son contexte et d'éclairer la pratique d'écoute du conte du point de vue de l'oralité première, relevant d'un acte de communication directe.

10 La communication directe se définit par l'acte de parole qui suppose que les acteurs, ici le public, le conteur et le conte, soient en interaction directe : il y a une immédiateté de la réception et de la réponse dans l'espace et dans le temps (Hymes, 2003). La prise en compte de l'aspect corporel et non verbal de cet acte n'est pas à négliger, comme les gestes ou le lieu. C'est cet aspect non verbal qui attire notre intention et notre intérêt ici :

Les émissions du corps, de quelque ordre qu'elles soient, n'ont de sens que si on les réfère à l'ensemble des données somatiques qui caractérise le groupe social où le sujet s'insère. Autrement dit, c'est l'ensemble des modalités corporelles du groupe social qui éclaire les significations qui traversent le corps de l'acteur. Il n'existe pas de naturel d'un geste ou d'une sensation.

(Le Breton, 1985, 47).

11 Comment percevoir et se rendre compte que les spect[acteurs], le public sont en train d'écouter? Quel(s) signe(s) apparent(s) nous montre(nt) que le public suit le fil de l'histoire? 


\section{Corps et posture d'écoute}

12 Analysons ce que nous désignons par "la posture d'écoute ", qui nous emmènera vers une autre dimension de cette analyse, l'atmosphère d'écoute qui, à son tour, nous conduira à nous interroger sur la circulation de la parole. Pour cela, décrivons et décortiquons la posture d'écoute telle qu'elle se révèle dans l'atmosphère particulière créée par le conteur dans la relation spécifique établie avec son public.

13 Lors des observations de terrain, le sentiment de ne "rien» percevoir, hormis des personnes assises, face à un conteur, était tout d'abord dominant. Était-ce dû à une intériorisation des mœurs somatiques de notre société telle que décrite par David Le Breton $(1985,47)$ qui montre comment, nous nous sommes construits une socialisation du corps relative à l'un des codes sociaux actuels : nous vivons aujourd'hui dans une configuration sociale qui s'est créée sur un rapport au corps et à l'intime spécifique mettant en avant une certaine importance de l'image (Le Breton, 1985, 36). Sans en avoir conscience, nous avions pourtant décelé des signes d'écoute à travers les attitudes des spectateurs. Des observations conduites lors de séances destinées aux enfants, le mercredi en bibliothèque, nous ont aidée à repérer ces signes.

La séance se déroulait dans une petite salle située derrière des rangées de livres avec une dizaine d'enfants accompagnés de leur nourrice ou de leurs parents. Au départ, les enfants s'étaient installés au fond de la salle, collés au mur ou à l'adulte qui les accompagnait. Tout le monde était assis par terre sur des tapis. Il n'y avait pas de chaises. Certains enfants avaient le pouce en bouche et quasiment tous se tenaient un peu recroquevillés. Timidité ? Peur de la conteuse ? Peur des autres personnes du public qui pouvaient sembler nombreuses à des enfants âgés de trois à six ans environ? La conteuse, avec quelques objets qu'elle présentait parfois au cours de ses histoires, a pris la parole. La séance a duré une demi-heure. Pendant ce temps, tout en écoutant les histoires, les enfants, petit à petit, se sont redressés, ceux qui avaient le pouce dans la bouche l'ont retiré. Ils ont quitté ensuite leur emplacement initial et se sont approchés progressivement et discrètement de la conteuse. Au départ, cette attitude n'était que très peu perceptible, puisqu'ils restaient assis, pour beaucoup, en tailleur. Mais à la fin de la séance, on pouvait remarquer qu'ils étaient tous assis en rond, tout près de la conteuse, à quelques centimètres d'elle, comme happés par les histoires, par les paroles. Les différentes observations réalisées auprès d'un public enfantin révèlent cette attitude de manière plus ou moins flagrante selon le lieu et l'installation (assis par terre, sur un tapis, ou sur des chaises et des bancs). La manière dont est installé le public peut favoriser cette attitude, ce déplacement vers le conteur.

Tout en poursuivant nos observations, nous nous sommes demandé si cette attitude, révélée chez un public d'enfants se retrouvait aussi chez un public d'adultes. Que dévoilait-elle ? Était-elle le signe de quelque chose de particulier?

Nous avons effectivement pu observer cette même attitude auprès du public adulte, mais de manière moins flagrante et plus discrète : les spectateurs adultes s'approchent eux aussi du conteur lors de l'écoute. C'est dans un bar où tous étaient assis par terre sur des tapis que nous l'avons observé d'une manière nette. Les spectateurs étaient, au début de la séance, au fond de la salle, assis en tailleur pour la plupart, un peu repliés sur eux-mêmes. Mais au fur et à mesure de la séance, ils se sont redressés tout en se penchant en avant, comme attirés par un aimant vers les conteurs. Ils s'en approchaient, doucement et lentement, de façon presque imperceptible. À la fin de la 
séance, comme chez les enfants, les spectateurs n'étaient plus qu'à quelques centimètres des quatre conteurs : penchés en avant, le dos bien droit, inclinés vers eux, comme s'ils s'approchaient au plus près des paroles, du récit, attirés vers les mots qui sortaient de la bouche de chacun des conteurs qui racontaient tour à tour une histoire.

Dans les lieux où les spectateurs étaient assis sur des chaises, nous avons également remarqué cette tendance à s'approcher, mais de manière plus discrète.

Avant que le conteur arrive sur scène et commence à conter, les spectateurs, en position d'attente, ont tendance à être "avachis » au fond de leur siège. Lorsque le conteur est là, ils se redressent légèrement. Puis, au fur et à mesure du déroulement de la séance, nous avons remarqué une similitude dans l'attitude du public: les spectateurs se redressent discrètement, penchent leur buste en avant, accompagnant ce mouvement de la tête qui s'avance, légèrement inclinée vers le conteur, comme s'ils lui offraient cette partie de leur corps, leurs oreilles se plaçant ainsi au plus près des paroles entendues, et les yeux grand ouverts. Parfois, à la fin de certaines séances, nous avons pu voir que les chaises avaient été déplacées, silencieusement, de quelques centimètres en direction du conteur. Soazig Hernandez souligne, d'un autre point de vue, ce phénomène :

[...] le conteur doit se trouver en général au centre d'un demi-cercle d'auditeurs. Si ce cercle n'est pas créé par une scène ou des chaises, les spectateurs ont tendance à le créer spontanément et se positionnent «tout naturellement » en arc de cercle autour du conteur. Il est important de souligner que cette mise en espace est un rituel récurrent et essentiel des séances de conte (...) qu'il s'agisse de spectacles à l'intérieur ou à l'extérieur.

(Hernandez, 2005, 197).

19 Cette attitude, nous la nommerons "posture d'écoute ", une posture qui n'est pas que corporelle, mais aussi physique et mentale.

La relation avec le conteur prend une double dimension: celle du corps et celle de l'esprit. Le spectateur, pour être attentif, adopte une disposition corporelle qui est le signe d'une disposition mentale d'écoute. Nous comprenons alors que le spectateur, contrairement à l'impression de passivité qu'il donne de prime abord, ne se pose pas comme un simple réceptacle d'histoires.

21 Il y a comme une forme de paradoxe dans cette posture : tandis que l'attitude du corps est d'apparence " passive », l'esprit est " embarqué ». C'est bien là le signe que le public adopte une posture d'écoute. Sommes-nous ici en présence d'un code social intériorisé ?

C'est une symbolique intériorisée à tel point qu'elle se confond à la chair du sujet qui permet à celui-ci d'appréhender et de déchiffrer les innombrables stimuli qu'il reçoit en permanence du monde extérieur (ou de son propre corps). La connaissance corporelle que le sujet possède de son environnement se trouve dans ses symbolismes, ses contours, ses limites, et sa tonalité. Il n'y a de possibilités de perception et d'action sur le monde que parce qu'il y a un ensemble d'éléments significatifs qui permet à l'organisme de classer la profusion des informations qu'il reçoit à chaque instant.

(Le Breton, 1985, 41).

Ces observations nous révèlent le point de départ d'une activité peu visible et peu perceptible, si l'on cherche à en donner des conclusions trop hâtives, où ces éléments extérieurs nous signifient qu'il y a une activité interne qui passe par le tissu corporel. En cela le jeu de mots entre spectateur et acteur et l'emploi du terme "spect[acteur] » prend tout son sens. Mais, qu'est-ce qui se joue à l'intérieur? Comment et à partir de 
quels éléments externes aux spect[acteurs] cette intériorité est-elle créée ? Autrement dit, quelle relation est établie avec le conteur pendant le temps d'écoute du conte? Et, au-delà de cette posture, y a-t-il d'autres codes à l'œuvre dans l'écoute du conte?

Poursuivons l'analyse à travers un autre élément : l'atmosphère d'écoute. Nous venons de voir ce qui se passe du côté des spectateurs. S'il existe une posture spécifique de la part des spect[acteurs], c'est bien parce qu'ils entrent en relation avec le conteur qui leur propose une histoire. Et même si nous tentons d'appréhender le point de vue du public, il ne faut pas pour autant nier la personne du conteur.

\section{Corps, parole et atmosphère d'écoute}

La posture d'écoute est une forme d'expression corporelle, certes d'apparence passive, mais qui révèle un aspect qui est de l'ordre de l'invisible : l'atmosphère d'écoute. Nous avons conscience que l'emploi de ce terme peut paraître subjectif, cependant, il révèle un état de l'esprit qui est spécifique à cette pratique culturelle. En effet, le corps se mobilise pour permettre que tout se joue à l'intérieur. Intervient ici l'action du conteur : il va permettre au public d'entrer dans une forme de relation avec lui. À cet égard, sommes-nous face à une forme de socialisation latente du corps ${ }^{7}$ ? À cette fin, une atmosphère propre et particulière à la pratique du conte va être créée, au sein de laquelle le conteur va proposer, à travers des codes sociaux, un échange, une interaction que l'on retrouve spécifiquement dans cet art. Mais quels sont-ils? Vers quoi ces codes renvoient-ils? Comment intervient ici le conteur?

Par atmosphère d'écoute, nous entendons ici une ambiance dans laquelle le spectateur se laisse emporter, immerger dans l'histoire, le conte. Cette atmosphère se crée dans un cadre (Goffman, 1991); elle s'est présentée dans nos observations ainsi que dans le discours des enquêtés.

Ce sentiment ou cette impression s'exprime dans les entretiens au cours desquels, rapidement, la notion d'atmosphère ou « d'ambiance » apparaît. Il semblerait que nous soyons ici confrontés à un signe permettant de comprendre que les enquêtés sont " entrés » dans l'atmosphère d'écoute : ils sont " embarqués ». Quels sont ces éléments qui permettent de se laisser happer par les paroles du conteur?

Il apparaît que le lieu a une certaine importance : il permet de participer à la création de cette ambiance. On peut aussi souligner sa dimension extra-ordinaire : il permet de " couper » avec les activités quotidiennes.

Y aurait-il, dans le passage d'un lieu où se déroulent les activités quotidiennes à un lieu extérieur au quotidien, un glissement à partir duquel le spectateur effectue une séparation mentale? Le décor, même sobre, participe à la mise en scène dans l'espace permettant d'embarquer l'auditeur dans l'écoute.

Un élément n'appartenant pas directement au décor, mais de plus en plus pris en compte dans les salles de spectacles, est l'assise, le fait d' « être bien installé ", il semble majeur dans l'adoption de la posture d'écoute. Les autres éléments du décor ont aussi leur rôle, ce sont eux qui vont permettre ce glissement et participer à sa mise en place. À commencer par la lumière qui est mieux appréciée lorsqu'elle est tamisée. Contribuant à créer une ambiance d'écoute, elle installe les spectateurs dans un « cocon » qui les protège du monde extérieur. Cette luminosité s'accorde à un nouvel éclairage, celui qu'offre le conteur à travers les histoires qu'il propose et raconte. La 
posture d'écoute adoptée par le spectateur est bien le signe qu'il s'est ouvert vers cette dimension à laquelle le conteur l'invite. Il semblerait que le corps du spectateur tende à se cristalliser, à créer une forme de socle, à partir duquel il va fixer son attention vers l'espace de parole que proposera le conteur au cours de ses histoires : sommes-nous ici face à une "dialectique du caché et du manifeste» (Bachelard, 2004, 110)? Gaston Bachelard nous en propose la définition suivante :

L'être qui se cache, l'être qui « rentre dans sa coquille » prépare une " sortie ». Cela est vrai sur toute l'échelle des métaphores depuis la résurrection d'un être enseveli jusqu'à l'expression soudaine d'un être taciturne. En restant encore au centre de l'image que nous étudions, il semble qu'en se conservant dans l'immobilité de sa coquille, l'être prépare des explosions de l'être temporel, des tourbillons d'être.

(Bachelard, 2004, 110).

Par l'adoption d'une posture d'écoute, le spectateur s'ouvre-t-il vers une forme de vécu du temps spécifique? Il semblerait que oui, au regard de certains termes employés par les personnes interrogées : « être embarqué », « être emporté ", « être ailleurs ».

Cet « être ailleurs » est-il le signe de l'embarquement vers une dimension particulière de l'écoute? Il ressort que la construction d'une atmosphère, insérant le spectateur dans un cocon, lui permet de s'ouvrir à une dimension de l'extraordinaire, qui est celle qu'offre le conte oral.

Cependant, cette atmosphère ne se crée pas seulement en raison du lieu qui place les acteurs hors de leurs activités quotidiennes. Elle est également mise en place par l'intervention du conteur qui permet au spectateur de vivre cette dimension de l'extraordinaire. Quelle relation s'établit alors entre lui et le public? Quelle place est investie par le conteur? Comment s'inscrit-il comme un élément majeur dans la création de cette atmosphère?

Le conteur met en place une relation qui passe par le langage du corps, c'est-à-dire la gestuelle, et par un autre support, la parole :

Lors de la transmission de l'histoire, le conteur joue sur des informations extratextuelles, un jeu d'approche, de provocation pour faire entrer l'auditeur dans l'espace du conte. La lenteur, la vitesse de la voix, le silence, tour à tour se chargent de sens. Le texte mis en son est transmis en consonance avec d'autres langages. (Hernandez, 2005, 165).

C'est l'image du " conteur-passeur ", exprimée par Anne-Sophie Haeringer (2006), qui illustre cette dimension où le conteur, d'une certaine manière, s'efface derrière le conte, "de sorte que le conte seul agisse». Pour ce faire, le conteur ouvre sur un espace-temps de la fiction créé avec - notamment- l'emploi de formulettes, qui annoncent le début et la fin du conte :

[...] qui installe en un temps et un lieu des personnages auxquels il arrive toujours quelque chose, il se pose a priori comme une forme close sur elle-même, dont le début et la fin soulignent qu'ils nous font entrer dans la fiction ou nous en congédient.

(Carlier, 1998, 8).

L'atmosphère d'écoute est donc un espace de fiction, une séparation d'avec le réel ${ }^{8}$. Elle est l'objet d'une fabrication, d'une création offerte par le conteur au public. Ainsi, le public va positionner et légitimer l'artiste qu'est le conteur. Ce dernier installe une atmosphère et emmène le spectateur dans le temps de la fiction où le «logos », le discours de la rationalité, laisse place au muthos, celui qui ouvre sur l'irrationnel, l'irréel. 
Nous voyons donc qu'un ensemble d'éléments insère la pratique du conte oral dans une situation particulière d'énonciation, de performance (Baumgardt et Derive, 2008, 52) qui installe les acteurs en présence - auditeur et conteur, énonciateur et destinataire dans un temps et un espace particulier, s'inscrivant hors de l'ordinaire, et qui met en jeu la dimension de la corporéité. Cette disposition des corps et des esprits leur permet d'entrer dans une relation sociale spécifique. Nous avons également remarqué à plusieurs reprises que cette disposition spatio-temporelle permet aux spectateurs d'être embarqués. Mais, quels sont les termes de cette relation? Autrement dit, dans quelle mesure, cette circulation de la parole permet-elle aux acteurs en présence d'être dans une relation d'échange?

\section{Entre don et immatérialité, le conte oral comme objet d'échange}

\section{De la performance au don : circulation de la parole}

En quoi et comment la pratique actuelle du conte oral telle que l'on peut l'observer en France participe-t-elle d'un échange par le don s'insérant dans un fait social complet (Mauss, 1923-1924)?

Dans quelle mesure existe-t-il un contrat dans cette relation? S'il existe, quel est-il ? Que révèle-t-il ? Et dans quelle mesure cette relation présente-t-elle des formes de transmission? Nous nous appuierons sur les travaux de Maurice Godelier (1996) et de Marcel Mauss pour apporter des éléments permettant de saisir les enjeux à l'œuvre dans cette relation d'échange.

39 La pratique du conte oral, d'apparence passive du point de vue de l'auditeur, nous fournit des éléments d'approche et de compréhension du fait qu'il ne s'agit pas d'une pratique figée, mais bien d'une relation, où le spectateur participe, dans laquelle il existe une dynamique, une rythmique codée socialement. Dépasser cet a priori d'un aspect figé nous semble avoir son intérêt pour sa compréhension. Nous nous référons à ce déplacement du regard évoqué par Marcel Mauss $(1923-1924,103)$ dans ses conclusions.

Pour introduire la question du don relative à notre exemple, peut-on dire qu'il y a un préalable de l'auditeur $^{9}$ ? Il se déplace, donne de son temps, inscrit l'événement dans son agenda, invite peut-être des personnes (ami-e-s, collègues, famille, conjoint-e, etc.), réserve et paye peut-être une place. Il se produit une éventualité d'actions et/ou d'intentions, d'émotions, en amont de sa venue auprès du conteur. De même, le conteur ne réalise-t-il pas un don préalable à celui des auditeurs? Ce que l'on constate, c'est qu'il existe une relation d'échange par le don dans le cadre de la pratique du conte oral et de son écoute. Cependant, qui, de l'auditeur ou du conteur, donne en premier? Comment est engagée, ou s'engage, cette relation sociale directe et orale?

41 En analysant la morphologie de l'écoute chez les auditeurs, nous avons adopté un nouveau regard sur leur attitude et sur cette codification, notamment corporelle, relative à un fait social installant l'échange du conte entre le public et le conteur.

En considérant que le conte est l'objet d'un échange, où « ce qui est donné est unique, non reproductible et collectif, résultant d'une synthèse opérée dans un contexte et à un moment précis par une personne délivrant à son auditoire le produit d'un travail, lui- 
même fondé sur un don antérieur » (Bru, 2012, 99), dans quelle mesure ce conte va-t-il être reçu et rendu? Nous sommes dans une configuration où le conteur peut s'adapter aux réactions du public, jouer avec ses réactions par différents procédés, comme provoquer la peur, la surprise, etc. N'est-ce pas le signe que l'auditoire donne son attention? Dans cette mesure, le conteur ne reçoit-il pas une attention à la fois individuelle et collective avant de donner son conte ? Est-ce là le signe que nous sommes face à une forme de don/contre-don qui paraît s'ajuster en direct ? Il y a là une tension qui s'exerce, le conteur avec son corps, sa parole et son conte va conformer de manière directe sa façon de raconter pour tenir le public en haleine.

Cependant, avant de donner son attention, le public ne reçoit-il pas la présence du conteur? L'analyse précédente sur l'attitude des corps semble en attester. S'il donne, cela montre, si nous nous référons au système d'échange par le don dans une conception maussienne, qu'il a reçu de manière à la fois individuelle et collective, puisque le conteur s'adresse à un ensemble (Esquenazi, 2003,4) - tout en ayant la possibilité à un moment, peut-être le temps d'un instant, de s'adresser en particulier à une personne de l'auditoire. Il y aurait une sorte d'équilibrage du don qui serait à la fois individuel et collectif, à la fois en soi, pour soi, pour l'autre et pour l'ensemble. Un conte ne peut être donné qu'une fois de la même manière ${ }^{10}$. Le conteur, ne serait-ce que par sa gestuelle, son corps, ne racontera pas de la même manière face au même ensemble, dans un lieu différent, dans une acoustique différente, selon le moment de la journée. L'histoire ne pourra donc pas être re-donnée de la même façon, puisque le rendu suppose implicitement une forme d'intérêt, une valeur ajoutée, parce qu'il existe des variations du contexte à chaque énonciation. Mais quelle est cette valeur ajoutée ?

Cette interrogation nous conduit à une autre dimension du don évoquée par Marcel Mauss (1923-1924, 16) : «Ce qui, dans le cadeau reçu, échangé, oblige, c'est que la chose reçue n'est pas inerte ». Cette dimension, c'est sa valeur symbolique :

La valeur de l'objet, c'est la puissance qu'il a en lui comme la puissance qu'il peut donner à des individus ou à des groupes dans des rapports qu'ils veulent avoir ou qu'ils nouent avec d'autres.

(Bert, 2007, 4).

45 À ce titre, une des critiques faite à Marcel Mauss est relative à la valeur symbolique de l'objet. Il convient de l'aborder étant donné que, sans cette dimension, nous ne pourrions apporter qu'une analyse abstraite du fait qui nous interroge. Marcel Mauss écrit que la chose échangée n'est pas inerte. L'explication qu'il en donne a été critiquée par Claude Lévi-Strauss ${ }^{11}$ et autrement argumentée par Maurice Godelier $(1996,44-49)$. Il s'agit de la quatrième obligation (relative aux dieux et aux offrandes) s'intriquant avec le rapport au symbolisme et à l'imaginaire :

Or, l'imaginaire n'a de force que quand il est croyance, norme de comportement, source morale. Et nous comprendrons bientôt que c'est précisément cette force de la croyance en l'imaginaire que Lévi-Strauss a occultée en affirmant la primauté du symbolique sur l'imaginaire.

(Godelier, 1996, 47).

Nous savons aujourd'hui que le symbolisme, tout comme l'origine du langage, est apparu progressivement. L'apparition du langage articulé chez l'humain s'est réalisée, en partie, à partir d'une disposition du corps, celle de l'abaissement du larynx. La station debout a permis ce changement (Warnier, 1999) jouant en faveur du développement de la double articulation du langage. 

capacité à symboliser? Nous rejoignons ici le postulat de Maurice Godelier. La capacité de symboliser et l'apparition du langage articulé sont effectivement des produits sociaux spécifiques à l'espèce humaine, mais qui sont apparus à travers le temps. Cet aspect du symbolique nous intéresse ici, car il va nous permettre d'aborder la relation à l'imaginaire opérée lors de l'écoute des contes par le public.

\section{Donner à percevoir, donner à imaginer ou de l'immatérialité d'une relation orale}

Le conte oral implique autre chose qu'une simple réception où le spectateur est un acteur social s'impliquant à sa manière. Il peut donc être considéré comme un acte volontaire (Godelier, 1996, 21). Le public lui ayant comme donné la parole par son attention, le conteur peut alors ouvrir un espace de parole. Serait-ce une obligation de rendre cette attention en embarquant alors son public hors de l'ordinaire? Serait-ce le terme d'un contrat (Godelier, 1996,22) engageant public et conteur? Le public sollicitant l'artiste installe une demande de partage du conte, plaçant ainsi le conteur dans une forme de hiérarchie où non seulement il connaît l'histoire, mais a appris à la restituer. Cette position hiérarchique peut être envisagée à partir du point de vue selon lequel, sans public, un artiste pratiquant un art vivant ne peut se passer d'un auditoire : autrement, quelle serait la mesure de son hexis social? Nous constatons donc qu'une forme de solidarité et de partage existe dans les termes de la relation entre le public et le conteur.

Examinons un moment le point de vue selon lequel une hiérarchie placerait les écoutants en position de supériorité (Godelier, 1996, 22) vis-à-vis du conteur. La dette du conteur ne serait-elle pas d'embarquer l'auditeur dans le conte? Mais quelle dimension dévoile ce terme embarqué?

Il s'agit d'un temps où un corps accompagnant une parole déroule une histoire - peu importe l'histoire. Ce qui nous interroge ici est ce qui se joue entre l'énonciateur et le destinataire du conte. Cette parole sera visualisée, à la fois par le conteur et par l'auditeur dans le terme d'une communication sociale directe. Le temps du conte, tous et chacun seront dans une sorte de voyage immobile où tout se joue de l'intérieur. Ce voyage consiste en la fabrication d'images mentales que le conteur donne à voir à l'auditeur. Être embarqué, c'est donc être dans une relation sociale de l'imaginaire ${ }^{12}$, dont le premier résultat est une visualisation, une fabrication d'images mentales, que ce soit chez celui qui raconte ou chez celui qui écoute. Cet embarquement est le signe intérieur que l'auditeur est dans l'espace de l'écoute.

51 Une des particularités de cette opération mentale est que chaque image est différente d'une personne à l'autre. Le conteur donne à percevoir une image. Cette image perçue relève de l'esprit ${ }^{13}$, une image partagée à la fois individuelle et collective ${ }^{14}$.

52 Définir ce qu'est être embarqué nous permet de comprendre que l'écoute du conte ne s'apparente pas uniquement à un échange par le don: il existe aussi une relation de transmission (Godelier, 1996, 53) relevant de l'immatériel. Le conteur, par l'action de conter, transmet son conte à l'auditoire :

- de façon collective, étant donné que tous écoutent dans le même temps, 
- de façon individuelle, étant donné que chacun visualisera à sa manière et retiendra différemment les éléments du conte, qu'il s'agisse de la trame ou de détails. Le simple exercice qui consiste à demander à au moins deux personnes différentes de raconter un même conte qu'elles ont entendu lors de la même « racontée » le démontre.

53 Nous voyons ici que nous sommes dans le symbolique (Godelier, 1996, 35-36), entendu dans un double sens, un moyen, une relation de communication qui passe par le langage et qui s'inscrit dans les termes d'un accord. Cet accord relève notamment du fait que tous les acteurs de cette relation savent et reconnaissent que le conte ne dit pas le vrai : ce temps est le temps du muthos où l'on pourrait qualifier le conteur de menteur, parce que l'on sait que l'histoire qu'il raconte n'est que pure fiction, et pourtant, il existe une adhésion des auditeurs.

Nous remarquons qu'il ne s'agit pas d'un simple échange, mais que l'écoute du conte relève d'une forme de croyance ${ }^{15}$ : le public croit à l'histoire durant tout le temps de l'écoute. Ainsi, le public donne-t-il une forme de pouvoir au conteur: celui d'embarquer les auditeurs l'instant de la racontée, simplement par sa voix et sa gestuelle. Sans nul autre artifice, il va faire voyager les esprits dans un autre temps et un autre espace. Le conteur aurait-il un rôle, un statut spécifique?

55 N'y aurait-il donc pas comme une relation de pouvoir: pouvoir de donner un conte, pouvoir de l'écouter, pouvoir de le recevoir, pouvoir de le restituer. Nous sommes ici dans l'esprit des choses, de la pensée. Il semblerait, en allant au-delà de l'analyse de Maurice Godelier, que le conte ne relève pas simplement du don et d'une transmission de la part du conteur et à l'adresse du public. Il y a autre chose. Mais quelle est cette chose?

Cette chose relève de la croyance et du pouvoir conférés au conteur. En effet le conte oral, bien qu'il instaure une relation d'échange par le don et implique une forme de transmission, a une autre spécificité liée à la représentation qu'on a du conteur. Ce dernier a le pouvoir d'embarquer un ensemble de personnes dans des pays de Cocagne, d'emporter légitimement le public vers un ailleurs immatériel. C'est cette immatérialité qui nous intéresse, car elle implique autre chose qu'un simple don :

D'ailleurs, dans beaucoup de sociétés, et, comme on le sait, au moins depuis Marcel Mauss, le pouvoir n'est pas toujours lié à l'argent, il est d'abord lié au prestige, à la connaissance mystique, c'est-à-dire à celle des initiations... L'autorité d'un individu ne vient pas forcément de la richesse, mais vient d'autre chose, que l'on appellerait, nous, « l'immatériel ».

(Bert, 2007, 5).

57 Le statut d'artiste du conteur lui confère une position spécifique liée au prestige. Ce prestige est conséquent d'une représentation, d'une croyance de la part des auditeurs ${ }^{16}$ selon laquelle le conteur est un initié : un pouvoir, un savoir-faire lui ont été transmis ou il les a acquis, lui conférant son autorité. Nous rejoignons là les travaux de Marcel Mauss :

En tout ceci, il y a une série de droits et de devoirs de consommer et de rendre, correspondant à des droits et des devoirs de présenter et de recevoir. Mais ce mélange étroit de droits et de devoirs symétriques et contraires cesse de paraitre contradictoire si l'on conçoit qu'il y a, avant tout, mélange de liens spirituels entre les choses qui sont à quelque degré de l'âme et les individus et les groupes qui se traitent à quelque degré comme des choses.

(Mauss, 1923-1924, 19). 
observations confortent le discours commun. À l'évocation de leur statut, les acteurs mettent en avant la notion du don de l'artiste, comme s'il émanait d'une force supérieure, d'une inspiration extra-ordinaire, créatrice (Heinich, 2004, 83). Cette initiation l'inscrit dans une forme de compilation de savoirs acquis par et avec ses pairs avec obligation de la restituer en respectant les canons de son apprentissage et en cherchant des formes novatrices de présentation. Les possibles qu'ouvre ce statut sont multiples : restituer un acquis tout en innovant. La tradition est revisitée, réappropriée dans la perspective d'une nouvelle configuration sociale, dans un nouveau contexte. Nous voyons là l'aspect mouvant de la tradition, par ailleurs ancrée dans des contextes socioculturels précis.

La pratique d'écoute du conte renvoie à l'échange par le don s'articulant avec une dimension symbolique et immatérielle qui s'instaure et se joue dans l'imaginaire à l'œuvre lors des « racontées ». Cette littérature orale, re-nouvelée depuis les années 1970 en France, a emprunté de nouvelles formes en s'adaptant au contexte sociétal changeant. Quels que soient le lieu et l'époque, le conte sera adapté, le plus souvent dans le cadre d'une trame élaborée et peaufinée de bouche à oreille de conteur en conteur et de manière anonyme. La littérature orale est ainsi l'expression d'un imaginaire à la fois individuel et collectif, faisant œuvre dans la circulation d'une parole qui peut s'apparenter à une vibration rythmée et codifiée mettant en branle les corps et les esprits en présence lors de la performance qui donne au conteur son prestige. La "diction" du conte et son écoute sont de nos jours et dans nos sociétés souvent reléguées au monde des enfants et de l'enfantin, qualifiés de naïfs, mais la pratique actuelle est toujours porteuse d'enjeux, elle reflète la complexité sociale et suppose un échange sous forme de don. La transmission de ce qui est donné, et qui est une forme de l'immatériel, donne au conteur le prestige dont il jouit.

Est-ce en cela que nous pouvons parler de la «magie» du conte? Ce pouvoir symbolique conféré par une mise en œuvre collective dans la sphère de l'imaginaire et du conte vise-t-il à donner à penser le social ? L'écoute de la parole du conteur, ouvrant un temps et un espace hors de l'ordinaire, n'offre-t-elle pas de surcroît un espace de réflexivité à la fois individuelle et sociale? Ne s'agit-il pas en plus d'un don et d'une transmission du conteur à l'auditeur, d'une adresse de celui-ci au public afin qu'il exerce sa capacité à pouvoir rendre ${ }^{17}$ ?

\section{BIBLIOGRAPHIE}

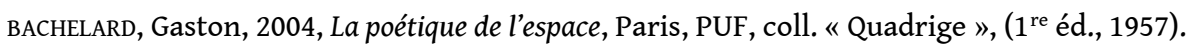

BAUMAN, Richard, 1975, Verbal art as performance, American Anthropologist, News Series, June, vol. 77, no 2, pp. 290-311, consulté le 23 mars 2015.

BAUMGARDT, Ursula et BORNAND, Sandra (dir.), 2009, Autour de la performance, Cahiers de littérature orale, $\mathrm{n}^{\circ} 65$.

Cahiers de littérature orale, 72 | 2012 
BAUMGARDT, Ursula et DERIVE, Jean (dir.), 2008, Littératures orales africaines, perspectives théoriques et méthodologiques, Paris, Karthala.

BERT, Jean-François, 2007, Entretien avec Maurice Godelier, Le Portique [En ligne], 19, mis en ligne le 15 décembre 2009, p. 1-5, consulté le 23 mars 2015.

BRU, Josiane, 2012, « Un renversement complet de la perspective » : le catalogue du conte populaire français à l'épreuve du terrain, Rabaska, Revue d'ethnologie de l'Amérique française, $\mathrm{n}^{\circ} 10$, p. 97-123.

CALAME-GRIAULE, Geneviève, 1965, Ethnologie et langage : la parole chez les Dogon, Paris, Gallimard, rééd. Lambert-Lucas, 2009.

CALAME-GRIAULE, Geneviève (dir.), 1999, Le renouveau du conte, Paris, CNRS Éditions.

CARLIER, Christophe, 1998, La clef des contes, Paris, Ellipses.

DIDIER, Béatrice, 1994, Dictionnaire universel des littératures, vol. 2 et 3, Paris, PUF.

ESQUENAZI, Jean-Pierre, 2003, Sociologie des publics, Paris, La Découverte, coll. « Repères ».

GODELIER, Maurice, 1996, L'énigme du don, Paris, Fayard.

GoffMAN, Erving, 1991, Les cadres de l'expérience, Paris, Éditions de Minuit, coll. « Le sens commun ».

HAERINGER, Anne-Sophie, 2006, Quand poser la question de l'énonciation c'est assurer la « relation », le « labo », lieu d'enquête sur la mise en œuvre du conte, Sociologie de l'art OpuS, novembre, $n^{\circ}$ 9-10, Paris, L'Harmattan, p. 179-194.

HALL, Edouard T., 1984, La danse de la vie, Paris, Seuil, p. 208.

HEINICH, Nathalie, SCHAEFFER, Jean-Marie, 2004, Art, création, fiction. Entre sociologie et philosophie, Paris, J. Chambon.

HERNANDEZ, Soazig, 2005, La mise en scène de l'œuvre conte, in Pascale Ancel, Catherine DutheilPessin et Alain Pessin (dir.), Rites et rythmes de l'œuvre, t. 2, Paris, L'Harmattan, coll. « Logiques sociales », p. 193-208.

HYMES, Dell, 2003, Models of the Interaction of Language and Social Setting, in Christina Bratt Paulston, G. Richard Tucker (dir.), Intercultural Discourse and Communication: The Essential Readings, Blackwell, pp. 13-53 [1st ed., 1967].

LE BRETON, David, 1985, Corps et société, essai de sociologie et d'anthropologie du corps, Paris, Méridiens Klincksieck, coll. « Sociologies au quotidien ».

LE BRETON, David, 1990, Anthropologie du corps et modernité, Paris, PUF, coll. « Sociologie d'aujourd'hui ».

MAUSS, Marcel, 1923-1924, Essai sur le don. Forme et raison de l'échange dans les sociétés primitives, L’Année Sociologique, seconde série, p. 30-186, consulté le 23 mars 2015.

MAUSS, Marcel, 1980, Sociologie et anthropologie, Paris, PUF, coll. « Quadrige » [précédé d'une « Introduction à l'œuvre de Marcel Mauss » par Claude Lévi-Strauss].

TENÈZE, Marie-Louise et DELARUE, Georges, 2000, Nannette Lévesque, conteuse et chanteuse du pays des sources de la Loire, Paris, Gallimard, coll. « Le langage des contes ». 
ROSSIGNOL, Émilie, 2008, Dans, à travers et autour du public adulte : pour une étude socioanthropologique du processus d'écoute du conte, mémoire de Master II, Université Pierre Mendès France, Grenoble 2.

TOUATI, Henri, 2000, L'art du récit en France. États des lieux, problématique, Étude réalisée à la demande du Ministère de la Culture (DMDTS) sous la responsabilité de l'AGECIF.

WARNIER, Jean-Pierre, 1999, Construire la culture matérielle, l'homme qui pensait avec ses doigts, Paris,

PUF.

\section{Sitographie}

Apac, Association professionnelle des artistes conteurs,

Centre des Arts du Récit en Isère,

CLiO, Conservatoire contemporain de Littérature Orale,

La Maison du Conte,

Mondoral,

Mythos,

Rncap, Réseau national du Conte et des Arts de la parole,

CASTA, Ange (réalisateur), FULCHIGNONI, Enrico (commentateur), BÂ, Amadou Hampâté

(participant), 1969, Entretien avec son Excellence Amadou Hampâté Bâ, émission « Un certain regard ", Office national de radiodiffusion télévision française (prod.), INA, consulté le 23 mars 2015.

\section{NOTES}

1. http://www.mondoral.org/

2. http://www.conteurspro.fr/

3. http://www.conte-artsdelaparole.org/reseau/

4. Le compte-rendu est accessible à l'adresse suivante: http://www.conteurspro.fr/ evenements3.html

5. Thèse en cours de préparation, sur la pratique actuelle du conte oral en France, sous la direction de Cécile Leguy (Université Sorbonne Nouvelle - Paris III). Voir aussi Rossignol (2008).

6. Nous emploierons ici le terme générique de conteur, prenant en compte aussi bien les conteurs que les conteuses.

7. Nous opposons le terme de socialisation latente à celui de socialisation manifeste comme, par exemple, celle exercée à l'école.

8. «Rien de plus écarté du réel que le conte : l'enfant et l'adulte l'admettent de concert dès que la voix a posé les cadres du récit » (Carlier, 1998, 9).

9. En considérant que l'objet de l'échange ne se limite pas au conte, mais peut être élargi à la relation qui est installée.

10. En référence aux propos de Nannette Lévesque rapportés dans l'appel à contribution de ce numéro (Tenèze et Delarue, 2000).

11. Cf. son Introduction à Marcel Mauss (1980).

12. «En se matérialisant dans des rapports sociaux, l'imaginaire devient une part de la réalité sociale » (Godelier, 1996, 42). 
13. Nous nous référons à Marcel Mauss qui précise que lors d'échange de biens matériels, une partie de l'échange relève de l'immatériel, de l'esprit. Il prend comme exemple celui des échanges chez les Maori, passim.

14. Ce qui distingue ici la création d'images mentales, par rapport à l'acte de lecture considéré ici comme un genre de la littérature orale et du spectacle vivant, et l'imaginaire qui peut en émerger, c'est que dans le cas de l'écoute du conte oral, l'histoire est reçue dans le même temps par chacun. Il y a une unité de la réception dans l'espace et dans le temps.

15. «[...] c'est de l'imaginaire que naissent les croyances » (Godelier, 1996, 42).

16. De la même manière, cette croyance se retrouve dans le cinéma, à l'issue du film où le spectateur, après s'être plongé dans l'ambiance du film, revient dans le temps du réel.

17. «La tradition orale, pour nous, c'est l'enseignement à tous les degrés » (Casta, 1969, 1'38).

\section{RÉSUMÉS}

Cet article présente une approche de la relation d'échange dans la pratique d'écoute du conte oral aujourd'hui en France. Le conte sera ici considéré à la fois comme un genre littéraire et comme une pratique sociale. Nous nous intéresserons tout particulièrement à la réception de cet art vivant.

Un bref aperçu du contexte et de l'émergence de la pratique actuelle du conte oral fera l'objet d'un premier temps. Nous proposerons ensuite une description qui sera interrogée du point de vue de la performance. Nous verrons comment l'écoute du conte place l'auditeur, le spect[acteur] dans une atmosphère d'écoute révélée par une posture spécifique qui concerne le public aussi bien que le conteur.

Nous chercherons enfin en quoi et comment cette écoute peut être renvoyée à une pratique d'échange par le don. Notre analyse s'appuiera sur les apports des travaux de Marcel Mauss et de Maurice Godelier pour montrer que l'écoute des contes englobe public et conteur dans une relation sociale qui ne relève pas seulement d'un don / contre don, et en quoi l'attitude du public permet-elle, avec la participation du conteur, d'élaborer une relation de l'imaginaire s'articulant avec d'autres éléments de l'immatériel.

This article presents an approach of the relation of the exchange in the case of the practice of the oral tale listening, today in France. It will be considered twice like a literacy gender and as a social practice. Beyond this living art, the aspect of the reception will interest us particularly.

A brief insight of the context and the emergence of this current practice will be the object of a first time. Then, a description of the listening practice of the tale will be proposed and interrogated as performance. We will see how the listening of the tale insert the auditor, the spect[actor], in a listening atmosphere revealed with a specific posture. This disposition brought into play public and storyteller.

Finally, we will search in what and how this listening is relative of a practice by gift exchange. This analysis will be based on the input of Marcel Mauss and Maurice Godelier research to demonstrate that listening tales includes public and storyteller in a social relation which not reveal also a gift/giving back and in what public attitude permit, with the participation of the storyteller, to elaborate a relation of the imaginary articulated with others elements of the immaterial. 
INDEX

Thèmes : anthropologie (Europe), littérature orale

Keywords : Gift, Storytelling, Listening Posture, Exchange, Audience

Mots-clés : don, contage, posture d'écoute, échange, auditoire

\section{AUTEUR}

ÉMILIE ROSSIGNOL

Oui’Dire Éditions 